\title{
Supply Chain Optimization of Assembly Manufacturer with Uncertainly Demand and Uncertainly Lead Time
}

\author{
ZHANG De-zhi, ${ }^{1, a}$, LI Shuangy-yan ${ }^{2, b}$, JIN Fang-ping ${ }^{1, c}$ \\ ${ }^{1}$ School of Transportation Engineering, Central South University, Changsha, 410075, \\ china \\ ${ }^{2}$ School of Transportation Engineering and Logistic, Central South University of Forestry \\ and Technology, Changsha, 410004, China \\ adzzhang@csu.edu.cn, ${ }^{b}$ lishuangyan585@sohu.com, 'king0315king@qq.com
}

Keywords: Uncertainly condition, Inventory control, Assembly system

\begin{abstract}
Order strategy optimization of multiple suppliers assembly manufacturing supply chain system under the random demand and random lead time is investigated in this paper. Manufacturers order strategy is sought for in order to minimize supply chain joint cost. Quantity ratio of different components due to assembly requirement is considered in optimization model. And scale benefits parameters of suppliers are used to reflect actual influence of the order's scale. Numerical example of two suppliers and one manufacturer supply chain is given to illustrate the order strategy.
\end{abstract}

\section{Introduction}

Researches on supply chain management with stochastic demand and variable lead time are becoming more recent years. Because the randomness and variableness make it difficult to predict orders accurately ${ }^{[1][2]}$. And with randomness and variableness, phenomenon of high inventory level, long turnover and out of stock will become common. Also they make it more difficult to get the proper inventory control ${ }^{[3-7] \text {. }}$

For assembly system, randomness and variableness make it impossible to control the inventory accurately specially. Because the negative effect of them have ripple effects. That is that the shortage of one component will lead to not only the production halts but also the accumulation of others, the consumption of any component will be restrained by the storage level of others.

\section{Problem description and Notation}

In this paper, we consider a two-stage supply chain with multi-suppliers and single manufacturer with the assumption that both lead time and demand are random variables. Each supplier provides just one kind of component, and the hypothetical component assembly proportion is 1: 1. The shortage of any component will make the assembly impossible. (Q, r) strategy is adopted by the manufacturer., as shown in Figure 1.

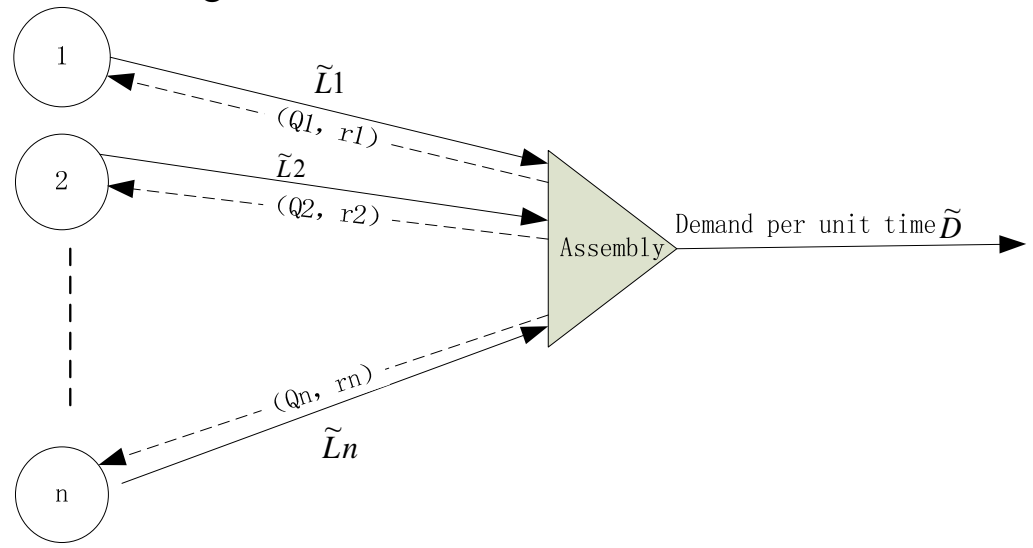

Fig. 1 Multi-suppliers and a single manufacturer supply chain 
Notations:

$i$ : index of suppliers

$s_{i}$ : order cost of component $i$ per unit time;

$\mu$ : mean of market demand per unit time;

$\widetilde{L_{i}}$ : actual lead time of component $i$

$\sigma$ : standard deviation of market demand per unit time

$\widetilde{X}_{i}$ : market demand during

$\beta$ : backorder coefficient

$h_{m}$ : unit inventory cost of component $i$ on the manufacturer per unit time

$A_{i}$ : setup cost of supplier $i$ per unit time

$\pi$ : shortage cost per unit time

$h_{i}$ : unit inventory cost of component $i$ on supplier per unit time

$[g]^{+}: \operatorname{Max}(g, 0)$

Decision variables

$Q_{i}$ : purchasing quantity of component $i$

$r_{i}$ : reorder point of component $i$

It can be known from the practice that the greater order quantity is, the more attention supplier pay to the order, and so more efficient corresponding services. With above assumption, lead time is variable, and related to the order quantity.

$$
\widetilde{L}_{i}\left(Q_{i}\right)=Q_{i}^{\theta} \widetilde{T}, \theta \geq 0
$$

Where $\theta$ indicates the extent of economies of scale ${ }^{[7]}$, which can be divided into two situations: $\theta=1$ means there's no economic scale benefit; and $\theta=0.5$ means that economic scale benefit is positive. Random variable $\widetilde{T}_{i}$ presents the unit lead time of component $i$. Lead time follows an exponential distribution with parameter $\lambda_{i}$. The demand of finished product follows a normal distribution $\left(\mu, \sigma^{2}\right)$. So the market demand of component during lead time is $\widetilde{X}=\widetilde{L} \widetilde{D}=Q_{i}^{\theta} \widetilde{T}_{i} \widetilde{D}$, whose mean value is given as Eq. (2).

$$
\mu_{\tilde{X}}=\int_{0}^{\infty} \int_{0}^{\infty} Q_{i}^{\theta} \tilde{T_{i}} \tilde{D} f\left(\tilde{T}_{i}\right) f(\tilde{D}) d \tilde{T}_{i} d \tilde{D}=Q_{i}^{\theta} \mu_{\tilde{D}} \mu_{\tilde{T}}=\frac{Q_{i}^{\theta} \mu}{\lambda_{i}}
$$

\section{Cost analysis of the manufacturer}

The total cost per unit time of the manufacturer is composed of components holding cost, shortage cost, and order cost. For component $i$, reorder point $r_{i}=\mu_{\widetilde{X}_{i}}+k_{i} \sigma_{\widetilde{X}_{i}}$, the average shortage of component $i$ is $B\left(r_{i}\right)$, abbreviations for $B_{i}$.

$$
B_{i}=E\left[\widetilde{X}_{i}-r_{i}\right]^{+}=\int_{0}^{\infty} \int_{0}^{\infty} \operatorname{Max}\left(Q_{i}^{\theta} \widetilde{T}_{i} \widetilde{D}-r_{i}, 0\right) f(\widetilde{D}) f\left(\widetilde{T_{i}}\right) d \widetilde{D} d \widetilde{T}_{i}
$$

When some component is in shortage, the assembly will stop, which will result in other components' accumulation. Every component's accumulation quantity should be calculated in materials holding cost. The average holding cost of component $i$ in manufacturer per unit time is as follows. 


$$
I_{i} \leq \frac{1}{2}\left[Q_{i}+\left(r_{i}-\mu_{\widetilde{x}_{i}}\right)+(1-\beta) B_{i}+\left(r_{i}-\mu_{\widetilde{x}_{i}}\right)+(1-\beta) B_{i}\right]+\sum_{j \neq i} \frac{\mu}{Q_{j}} B_{j}
$$

The shortage cost of the manufacturer caused by the shortage of component $i$ is $\frac{\mu}{Q_{i}} \pi(1-\beta) B_{i}$. And Ordering cost is $s_{i} \frac{\mu}{Q_{i}}$. So total cost of the manufacturer is given as follows:

$$
E C M=\sum_{i=1}^{n}\left(s \frac{\mu}{Q_{i}}+\frac{1}{2} Q_{i} h_{m}+k_{i} \sigma_{\widetilde{X}_{i}} h_{m}+(1-\beta) B_{i} h_{m}+\sum_{j \neq i} \frac{\mu}{Q_{j}} B_{j} h_{m}+\frac{\mu}{Q_{i}} \pi(1-\beta) B_{i}\right.
$$

\section{Cost analysis of supplier}

The cost of supplier $i$ includes two parts: setup cost and inventory cost. The length of product cycle is $T_{m}=\frac{Q_{i}}{\mu}$. So, the expected setup cost per unit time of supplier $i$ is $\frac{\mu}{Q_{i}} A_{i}$. Average material holding cost of supplier is $h_{i} \frac{Q_{i}}{2}$. The expected total cost per unit time of supplier $i$ is given as follows:

$$
\operatorname{ECS}_{i}=\frac{\mu}{Q_{i}} A_{i}+h_{i} \frac{Q_{i}}{2}
$$

\section{Joint cost of the two-stage supply chain}

The relationship between joint cost of whole supply chain and variable is given as Eq. (9).

$$
\begin{aligned}
& E C \leq E C M+\sum_{i=1}^{n} E C S_{i} \\
& =\sum_{i=1}^{n}\left(s \frac{\mu}{Q_{i}}+\frac{1}{2} Q_{i} h_{m}+k_{i} \sigma_{\tilde{X}_{i}} h_{m}+(1-\beta) B_{i} h_{m}+\sum_{j \neq i} \frac{\mu}{Q_{j}} B_{j} h_{m}+\frac{\mu}{Q_{i}} \pi(1-\beta) B_{i}+\sum_{i=1}^{n} \frac{\mu}{Q_{i}} A+h_{i} \frac{Q_{i}}{2}\right.
\end{aligned}
$$

\section{Solution Algorithm analysis}

As $\mu_{\widetilde{X}_{i}}$ is given by equation $r_{i}=\mu_{\widetilde{X}_{i}}+k_{i} \sigma_{\widetilde{X}_{i}}$ for $i=1,2 \cdots n$, when safety factor $k_{i}$ decided the reorder point is determined. And it is nonlinear relationship between the object function $E C$ and decision variable $k_{i}$ and $Q_{i}$ constrained by boundary limit. SQP algorithm is used to solute this model. In order to achieve the global optimal solution as far as possible, few initial points are start to obtain the local optimal solution, and then compare each partial solution, take the minimum as the objective function.

\section{Numerical example}

In this section, a supply chain with two suppliers and a manufacturer is considered. All parameters are selected randomly and 4 groups input parameters are tested .The parameters set satisfying the assumptions in this study. Backorder rate $\beta$ and order scale factor $\theta 1, \theta 2$ are changed to analysis the effect for supply chain of the backorder order scale. Then according to the above optimization model and its solving method, the calculation results were shown in table 1.

Table 1. optimization results with data set 1-4 


\begin{tabular}{llllllllll}
\hline $\begin{array}{l}\text { Parameter } \\
\text { value }\end{array}$ & Q1 & K1 & R1 & Q2 & K2 & R2 & $\begin{array}{l}\text { Cost of } \\
\text { supplies }\end{array}$ & $\begin{array}{l}\text { Cost of } \\
\text { manufacturer }\end{array}$ & $\begin{array}{l}\text { Joint } \\
\text { cost }\end{array}$ \\
\hline$\beta=1 \theta=1$ & 189 & 0.9 & 179.9 & 254 & 0.8 & 147 & 39.50 & 369.14 & 406.6 \\
$\beta=1 \theta=0.5$ & 333 & 0.95 & 17.7 & 381 & 0.85 & 12.03 & 22.59 & 341.7 & 3 \\
$\beta=0.5 \theta=1$ & 210 & 1 & 210 & 264 & 0.9 & 167 & 34.19 & 1075.86 & $\begin{array}{l}1110.3 \\
1\end{array}$ \\
$\beta=0.5 \theta=0.5$ & 402 & 0.95 & 19.6 & 427 & 0.88 & 12.94 & 19.33 & 426.32 & $\begin{array}{l}445.6 \\
5\end{array}$ \\
\hline
\end{tabular}

Note: $\theta=\theta 1=\theta 2$ in table 1

From the table1 and table2, it can be seen that the minimal joint cost per unit time is equivalent to 364.3. This is because that the backorder rate is a hundred percent, so the end customer's requirements can be delay to maximum limit, the reorder point $r$ is also reduced to maximum limit. And the greater order quantity is, the more economic scale benefit is.

\section{Conclusion}

This paper investigates supply chain optimization of assembly manufacturing consisting of multiple independent suppliers and a manufacturer with stochastic demand and variable lead time. It is can be seen if backorder rate and order scale effect, will change the total supply chain cost dramatically. The bigger backorder rate and order scale effect, the lower supply chain system cost.

\section{Acknowledgements}

This work was financially supported by National Social Science Foundation (No.11CGL032), Hunan Province Science and Technology Development Funds (No.2010FJ3007) and Freedom Explore Program of Central South University (NO.201012200100), Youth Science Foundation of Central South Forestry University of Science and Technology (No. 2009034B).

\section{References}

[1] Mohamed Aly Louly, Alexandre Dolgui, Faicel Hnaien: submitted to International Journal of Production Economics, 2008,115(1):236-247

[2] Dolgui, A: submitted to Automation and Remote Control 2001,62(12):2020-2026.

[3] Alexandre Dolgui, Mohamed-Aly Ould-Louly: submitted to International Journal of Production Economics,2002,78(2):145-152

[4] Dolgui, A.,Prodhon,C: submitted to Annual Reviews in control 2007(31):269-279.

[5] Zipkin P.H.Foundations of Inventory Management.McGraw-Hill,New York,2000:243-290

[6] Zhan Pang: submitted to Operations Research Letters,2011 39(5):375-379

[7] Metin Cakanyildirim, James H. Bookbinder, Yigal Gerchak: submitted to Int. J. Production Economics, 2000, 68: 217-228 